Journal of Geophysical Research: Solid Earth

\author{
RESEARCH ARTICLE \\ 10.1002/2013JB010604 \\ Key Points: \\ - Spatially anticorrelated secular accel- \\ eration pulses occurred in 2006 \\ and 2009 \\ - These pulses resulted in geomagnetic \\ jerks in 2003, 2007, and 2011 \\ - There is a standing wave at \\ core surface, not caused by \\ torsional oscillation
}

Correspondence to:

A. Chulliat,

chulliat@ipgp.fr

Citation:

Chulliat, A., and S. Maus (2014), Geomagnetic secular acceleration, jerks, and a localized standing wave at the core surface from 2000 to 2010, J. Geophys. Res. Solid Earth, 119 1531-1543, doi:10.1002/2013JB010604.

Received 13 AUG 2013

Accepted 5 FEB 2014

Accepted article online 8 FEB 2014

Published online 5 MAR 2014

\section{Geomagnetic secular acceleration, jerks, and a localized standing wave at the core surface from 2000 to 2010}

\author{
A. Chulliat ${ }^{1,2}$ and S. Maus ${ }^{2}$ \\ ${ }^{1}$ Institut de Physique du Globe de Paris, Sorbonne Paris Cité, Université Paris Diderot, UMR 7154 CNRS, Paris, France, \\ ${ }^{2}$ National Geophysical Data Center, NOAA, Boulder, Colorado, USA
}

\begin{abstract}
The geomagnetic secular acceleration (SA), defined as the second-order time derivative of the Earth's core magnetic field, is investigated using data from the CHAMP satellite. We present a set of SA spherical harmonic models calculated from 3 year time intervals of CHAMP data, centered on epochs ranging from 2002.19 to 2009.51 with a 30 day step. These models are parameterized as second-order Taylor expansions in time and are not regularized, except for SA degrees larger than 8 . We find that the SA underwent two power pulses in 2006 and 2009 at the core-mantle boundary (mostly on degrees 5 and 6) and at the Earth's surface (mostly on degrees 2 to 4). These pulses take the form of intense SA patches at the core surface in the low-latitude Atlantic sector and in the Indian Ocean sector. In the Atlantic sector, the 2006 and 2009 SA patches are markedly anticorrelated. Principal component analysis suggests that the two pulses are part of a standing wave of period about 6 years. At the Earth's surface, this wave results in a succession of geomagnetic jerks, i.e., sudden SA polarity changes, in 2003, 2007, and 2011 near the Atlantic sector. The 2011 jerk is detected using the latest observatory data available, including quasi-definitive data from January to October 2013. The origin of the wave is not clear; we find that it cannot be generated by the zonal toroidal flow of a torsional oscillation. Other possible interpretations are discussed.
\end{abstract}

\section{Introduction}

The observable part of the magnetic field generated within the Earth's outer core varies on time scales from 1 year to several tens of million years. Variations faster than 1 year are filtered out by the electrically conducting mantle. Variations between 1 year and a few centuries are well documented by continuous series of observatory records and, at the higher end of the observable spectrum, recent satellite missions. They include various manifestations of the geomagnetic secular variation (SV), such as the westward drift, the slow decay of the geomagnetic dipole, the growth of the low-field anomaly in the South Atlantic, and the drift of the North magnetic pole. The SV is caused by core flows, either convective or associated to magnetohydrodynamic waves, and diffusional processes [see, e.g., Finlay et al., 2010].

On subdecadal time scales, subtle variations in the SV are detected by calculating the second-order time derivative of the field, i.e., the secular acceleration (SA). At irregular time intervals, the SA at a given observatory undergoes sudden polarity changes, occurring in less than a year. These events are referred to as geomagnetic jerks [Courtillot et al., 1978] and separate time intervals where the SV varies almost linearly. Jerks have been widely investigated over the past three decades (see, e.g., Mandea et al. [2010], for a recent review), but the question of their origin remains unsolved. They can be either regional or global in their spatial extent, with a maximum time shift of 2 to 3 years between observatories. The most recent geomagnetic jerks were recorded in 2003 [Olsen and Mandea, 2007] and 2007 [Chulliat et al., 2010].

The Ørsted and CHAMP satellite missions have recently stimulated the research on the origin on geomagnetic jerks, by providing a global coverage of vector magnetic data of unprecedented precision and space-time resolution over more than a decade. Thanks to Ørsted and CHAMP data, it is now possible to calculate the SA from space. Spherical harmonic (SH) models from the CHAOS series [Olsen et al., 2009, 2010] and the GeoForschungsZentrum reference internal magnetic model (GRIMM) series [Lesur et al., 2010] have been used to derive global maps of the SA at the Earth's and core surfaces over the last decade. Using the CHAOS-2 model, Chulliat et al. [2010] showed that the total energy of the SA at the core surface peaked between 2005 and 2006 and that this energy was mostly contained within a large SA patch centered in the mid-Atlantic Ocean. They found that the increasing phase of this so-called "SA pulse" was causing the geomagnetic jerk observed at the core surface near 2003, while its decreasing phase was causing the 2007 
jerk. Using CHAOS-3, a more recent version of the same model, Silva et al. [2012] identified a 6 year periodic signal in the SA over the past decade, which, they claimed, is reminiscent of the recently discovered 6 year torsional wave within the core over the 1960-1982 time interval [Gillet et al., 2010]. It should be noted, however, that the SA from CHAOS- and GRIMM-type models should be handled with care, as these models rely on smoothing splines for representing temporal variations of spherical harmonic coefficients, following a well-established practice in the geomagnetic modeling community [see, e.g., Bloxham and Jackson, 1992; Korte and Constable, 2005; Finlay et al., 2012]. Taking the second derivative of splines can lead to spurious effects, even if the splines are of high enough order. In addition, in these models, splines are regularized, which can smooth the SA and lead to some attenuation effect near time interval boundaries.

In this paper, we report on the results of SA modeling over the last decade using an entirely different method, wherein the SA is modeled over a 3 year sliding window using second-order Taylor series. The modeling method is presented in section 2 . We then analyze the space-time characteristics of the obtained SA models (section 3 ) and their relationships with recent geomagnetic jerks (section 4). We find that another acceleration pulse occurred in 2009 and yet another jerk around 2011, using the most recent observatory data available, including quasi-definitive [Peltier and Chulliat, 2010] data from January to October 2013. Our analysis suggests the existence of a localized standing wave at the core surface over the last decade. In section 5, we discuss possible physical mechanisms that could explain such a wave; in particular, we show that this wave cannot be caused by zonal toroidal flows associated with torsional oscillations within the core.

\section{Secular Acceleration Modeling}

SA models were built from data acquired by the German CHAMP (Challenging Minisatellite Payload) satellite, from 1 January 2001 to the end of the mission in September 2010. We used a uniform data set (i.e., no data from other satellites or ground data) in order to avoid inconsistencies between data sets, which could appear as spurious features in the modeled SA.

Data processing prior to the inversion was performed along the lines described in Maus et al. [2010]. Here we briefly summarize this processing and point out a few differences or upgrades that were introduced with respect to that earlier publication. Data were first selected at the $20 \mathrm{~s}$ sampling interval then separated in two subsets: data at middle to low latitudes (from $-60^{\circ}$ to $60^{\circ}$ geomagnetic latitude) and data at high latitudes (above $50^{\circ}$ and below $-50^{\circ}$ geomagnetic latitude). In the overlapping region, the weights of the high-latitude data were tapered off to zero toward the low latitudes and vice versa. At middle to low latitudes, vector and scalar data from local times (LT) between 20:00 to 5:00 were considered; at high latitudes, scalar data at all LT were considered. Data were selected for periods of low geomagnetic activity, characterized by the Dst and am geomagnetic indices, the interplanetary magnetic field at the bow shock (interplanetary magnetic field (IMF) $B_{y}$ and IMF $B_{z}$ ), and the merging electric field $\left(E_{m}\right)$. Tracks were rejected when their along-track RMS residual with respect to the Pomme-6 model [Maus et al., 2010] was greater than $3 \mathrm{nT}$ and when they were surrounded by neighboring tracks having smaller RMS residuals. Data were then corrected for several effects: the misalignment between the magnetometer and the star tracker, the magnetic field generated by oceanic tides (using the model of Kuvshinov and Olsen [2005]), the magnetic field generated by plasma pressure gradient currents in the ionosphere (using the values obtained by Lühr et al. [2003]), and the magnetospheric magnetic field (using the model of Lühr and Maus [2010]). Data were weighted according to their local density on the sphere, in order to avoid overrepresentation of some areas with respect to others. More details including threshold values for data selection can be found in Maus et al. [2010].

For each model, the scalar potential of the internal magnetic field was described as a second-order Taylor series in time of spherical harmonic coefficients, in which the zeroth-, first-, and second-order coefficients would represent the main field (MF), SV and SA, respectively. Since a time-varying external field model was already subtracted from the data, only a minor correction of the external field was coestimated with the model. This correction accounts for inaccuracies in the baseline of the Dst index by a daily varying axial dipole in the solar magnetic reference frame. The maximum degree and order of the internal SH expansion was set to 30 for the MF and 15 for the SV and SA. Each SA model was built from a sliding window of 3 years of data, centered on epochs ranging from 2002.19 to 2009.51 , with a 30 day step. This method amounts to apply an implicit time averaging over 3 years and to use consecutive time windows that are uncorrelated in time. The window length was chosen as small as possible, in order to reduce the time averaging while 


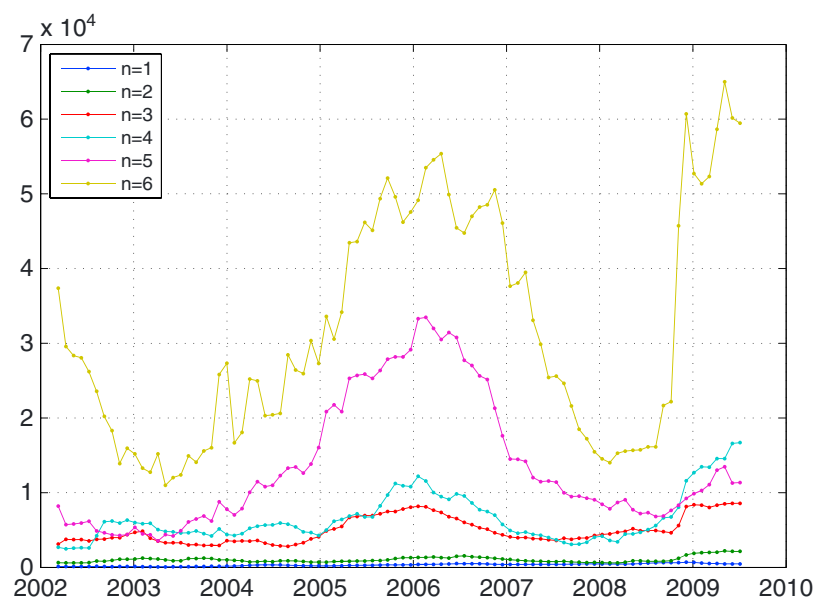

Figure 1. Time variation of the squared secular acceleration power for spherical harmonic degrees $n=1$ to 6 at the core-mantle boundary. Unit: $\left(\mathrm{nT} / \mathrm{yr}^{2}\right)^{2}$.

keeping the inversion stable. Model coefficients were obtained using a noniterative least squares method. Regularizations were applied to SV coefficients for degrees larger or equal to 14 and to SA coefficients for degrees larger or equal to 9 (by increasing the corresponding diagonal coefficients of the normal equations matrix to impose decreasing SV and SA spectra, see Maus et al. [2006], for details). In other words, the SH coefficients of degrees 1 to 8 were not regularized in any way.

\section{Secular Acceleration at the Core-Mantle Boundary}

The SA signal is very small at the Earth's surface. Typically, it amounts to only a few $\mathrm{nT} / \mathrm{yr}^{2}$ and can reach up to $20 \mathrm{nT} / \mathrm{yr}^{2}$ at some locations (not shown). This is about 10 times smaller than the SV, which can reach up to $200 \mathrm{nT} / \mathrm{yr}$ at some locations. At the core-mantle boundary (CMB), assuming the mantle electrical conductivity is negligible, the MF, SV, and SA are geometrically amplified by a factor of $(a / c)^{n+2}$, where $a$ is the Earth's radius, $c$ the core radius, and $n$ the degree of the $\mathrm{SH}$ expansion. For the MF, this amplification leads to a flat power spectrum, while for the SV and SA, the power increases with SH degree. It is thus difficult to provide an estimate of the order of magnitude of the SA at the CMB. It also means that the error on the smaller scales of the SA is greatly amplified at the CMB. In order to circumvent this difficulty, it is necessary to truncate the SA signal at some relatively low degree. In what follows, we relied on two approaches for extracting the meaningful SA signal at the CMB: either we truncated the SA at degree and order 6 or we filtered its $\mathrm{SH}$ expansion using a Hann window defined by $w(n)=(1+\cos (n \pi / N)) / 2$, where $N=10$. This selection was based on the realization that degrees above 6 seemed to contain many spurious features, while some interesting structures were seen in degrees smaller or equal to 6. The latter approach (Hann filtering) was used when plotting SA maps, in order to minimize ringing artifacts.

Figure 1 shows the time evolution of the degree-by-degree, unfiltered SA power, for degrees $n=1$ to 6 . As expected, the SA power increases with degree. There is a large pulse of SA centered on epoch 2006, in good agreement with earlier results from Chulliat et al. [2010], which relied on the CHAOS-2s model of Olsen et al. [2009]. However, compared to Figure 4 of that paper, the SA power for degrees $n=5$ and 6 is larger: about $3\left(n T / y^{2}\right)^{2}$ versus $1.9\left(n T / y^{2}\right)^{2}$ for $n=5$, about $5\left(n T / y^{2}\right)^{2}$ versus $1.8\left(n T / y^{2}\right)^{2}$ for $n=6$. This reflects the difference in the time averaging inherent in the CHAOS-2s modeling compared to the uniform 3 year time averaging of SA applied in our modeling. We also note that the 2006 pulse appears to be slightly positively shifted in time, by a few months, for degrees $n=5$ and 6 , with respect to the pulse obtained from CHAOS-2s. This is probably due to the applied temporal regularization in the CHAOS-2s modeling that is responsible for different smoothing times as a function of different spherical harmonic degrees and would thus smooth some of the peaks seen in Figure 1.

An even larger peak is conspicuous near 2009 for the degree $n=6$ of the power spectrum, reaching about $6\left(\mathrm{nT} / \mathrm{yr}^{2}\right)^{2}$. This second SA pulse could not be seen in results from CHAOS- $2 \mathrm{~s}$, as that model relied on data until March 2009 only. It is not seen either in degree-by-degree SA power spectra plotted from the more 

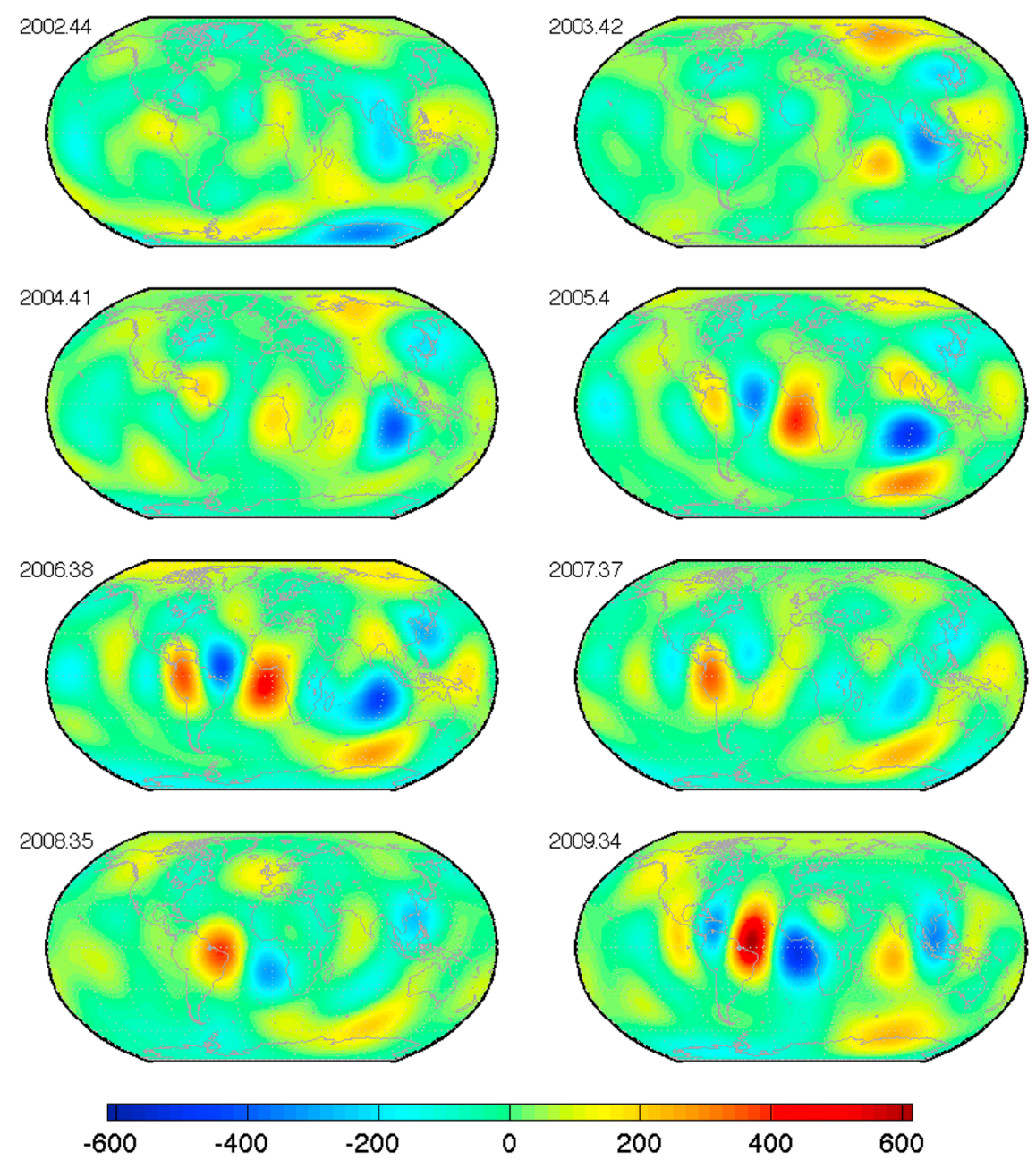

Figure 2. Secular acceleration maps (radial component) at the core-mantle boundary, from 2002.44 to 2009.34 , every 360 days, obtained from a Hann-filtered spherical harmonic expansion until degree $n=10$. Unit: $\mathrm{nT} / \mathrm{yr}^{2}$.

recent CHAOS-4 model (http://www.spacecenter.dk/files/magnetic-models/CHAOS-4/), although this model includes data until the end of the CHAMP mission (figure not shown here). The reason for this difference between our modeling and that of CHAOS-4 probably lies in the combined effect of SA regularization and the use of smoothing splines in CHAOS-4, which would be enhanced near the data set boundaries. Another pulse seems to occur near 2002 in our set of SA models (Figure 1); however, unlike for the 2009 pulse, this event apparently peaks outside the data set boundaries and occurs for SA degree $n=6$ only. For these reasons, we do not further consider this event and focus on the 2006 and 2009 pulses hereafter.

$S A$ maps at the CMB are displayed in Figure 2. Here we selected only one in every 12 models (i.e., roughly one model per year), to save space. Also, only the radial component is represented, as only this component is continuous through the CMB (because of the large difference in electrical conductivity between the core and the mantle) and is therefore directly relevant for core processes. The 2006 and 2009 pulses are conspicuous on the maps for epochs 2006.38 and 2009.34; they take the form of medium size SA patches at middle to low latitudes. We check that the SA patches in 2005.40 and 2006.38 are similar to those seen in Chulliat et al. [2010, Figure 3b] (which was plotted at epoch 2005); the main differences seem to be the slight shift in time already noted in Figure 1 and the lesser number of ringing artifacts, due to the Hann filtering of the model. In 2009.34, the three patches of alternate polarities located in the Atlantic sector have strikingly similar shapes as the 2006 patches in the same region and are of opposite polarities with respect to the 2006 patches. The SA in the central Atlantic patch is a bit more intense, reaching $600 \mathrm{nT} / \mathrm{yr}^{2}$ (to be compared with about $-400 \mathrm{nT} / \mathrm{yr}^{2}$ for the patch at the same location in 2006). The relationship between 2006 and 2009 
(a)

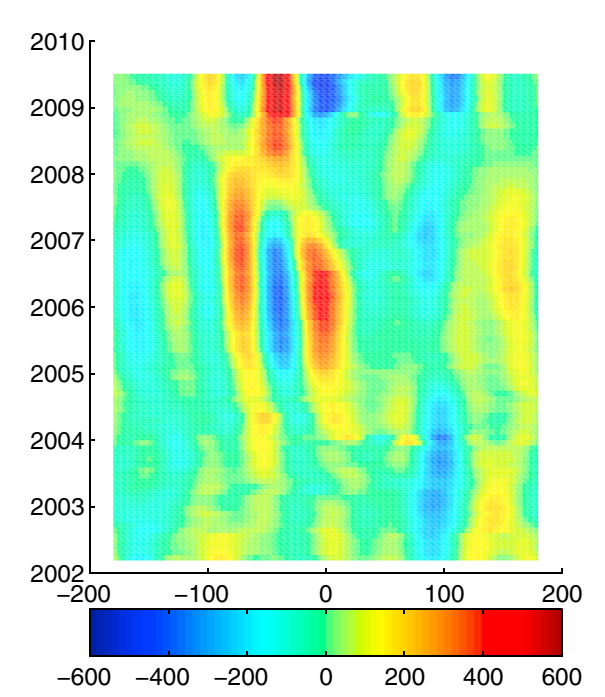

(b)

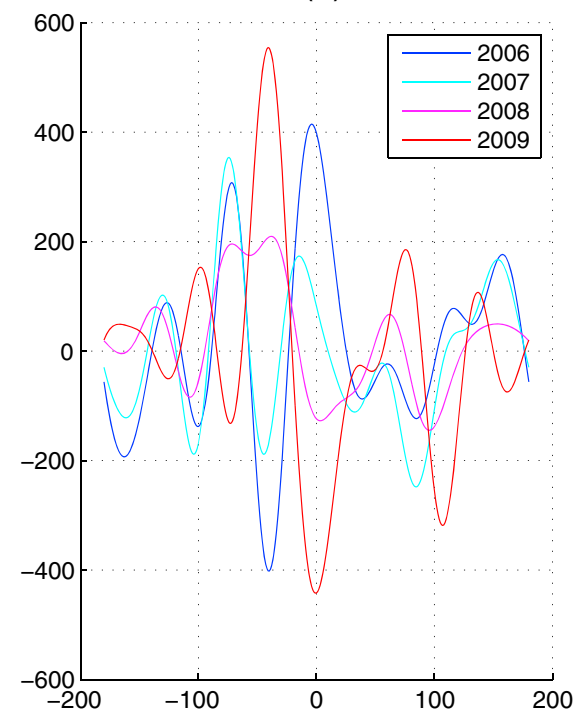

Figure 3. (a) Time-longitude diagram of the Hann-filtered secular acceleration, radial component, along the geographic equator at the core-mantle boundary. The longitude ( $x$ axis) is expressed in degrees, the time ( $y$ axis) in year, and the acceleration (color scale) in $\mathrm{nT} / \mathrm{yr}^{2}$. (b) Variation along the geographic equator of the Hann-filtered secular acceleration, radial component, at the core-mantle boundary for several epochs (same units).

patches at other locations is less clear, especially for the large and intense patches in the Indian Ocean sector. At all other epochs, the SA is smaller on average and does not reach values larger than $400 \mathrm{nT} / \mathrm{yr}^{2}$, in good agreement with the power spectra shown in Figure 1.

The apparent anticorrelation between the 2006 and 2009 SA patches in the Atlantic sector is further investigated using the time-longitude diagram along the geographic equator displayed in Figure $3 a$ (which was drawn from Hann-filtered SA coefficients). The 2006 and 2009 pulses are again conspicuous in this diagram; the three SA patches of alternate polarities in the Atlantic sector are seen at both epochs and reverse their polarities between 2006 and 2009. There is another, less intense, and nonreversing feature near $100^{\circ}$ longitude, to be associated with the northern Indian Ocean patch in Figure 2. The SA time-longitude diagram does not display any inclined feature typical of a propagating wave; on the contrary, the time-longitude diagram between 2005 and 2009.5 in the Atlantic sector is that of a standing wave.

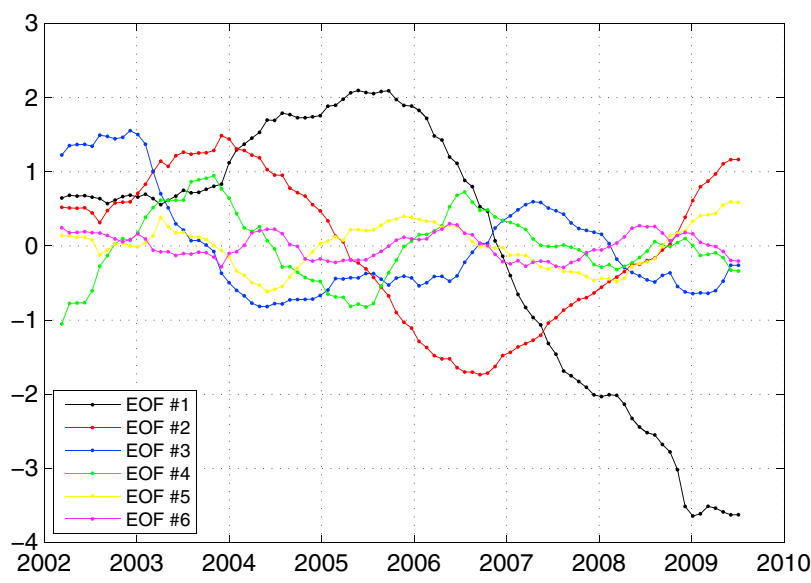

Figure 4. Principal component time series of the first six empirical orthogonal functions, describing $99 \%$ of the Hann-filtered secular acceleration over 2002.19-2009.51.
Figure $3 \mathrm{~b}$ shows in more detail how the 2006 and 2009 SA compare along the equator. The three patches of alternate and reversing polarities peak at longitudes $\Phi=-4^{\circ},-40^{\circ}$, and $-72^{\circ}$ in 2006 and at $\Phi=-1^{\circ},-41^{\circ}$, and $-73^{\circ}$ in 2009. The angular difference between each peak ranges from $\Delta \Phi=32^{\circ}$ to $\Delta \Phi=40^{\circ}$, which is consistent with $n=5$ $\left(\Delta \Phi=30^{\circ}\right)$ and $n=6\left(\Delta \Phi=36^{\circ}\right)$ sectoral modes, in good agreement with the power spectra shown in Figure 1.

The space-time structure of the SA signal can be better characterized using principal component analysis [see, e.g., Preisendorfer, 1988]. We analyzed the $90 \times 120 \mathbf{Z}$ matrix of $z(t, x)$ Hann-filtered SA model coefficients, where $t$ is the 

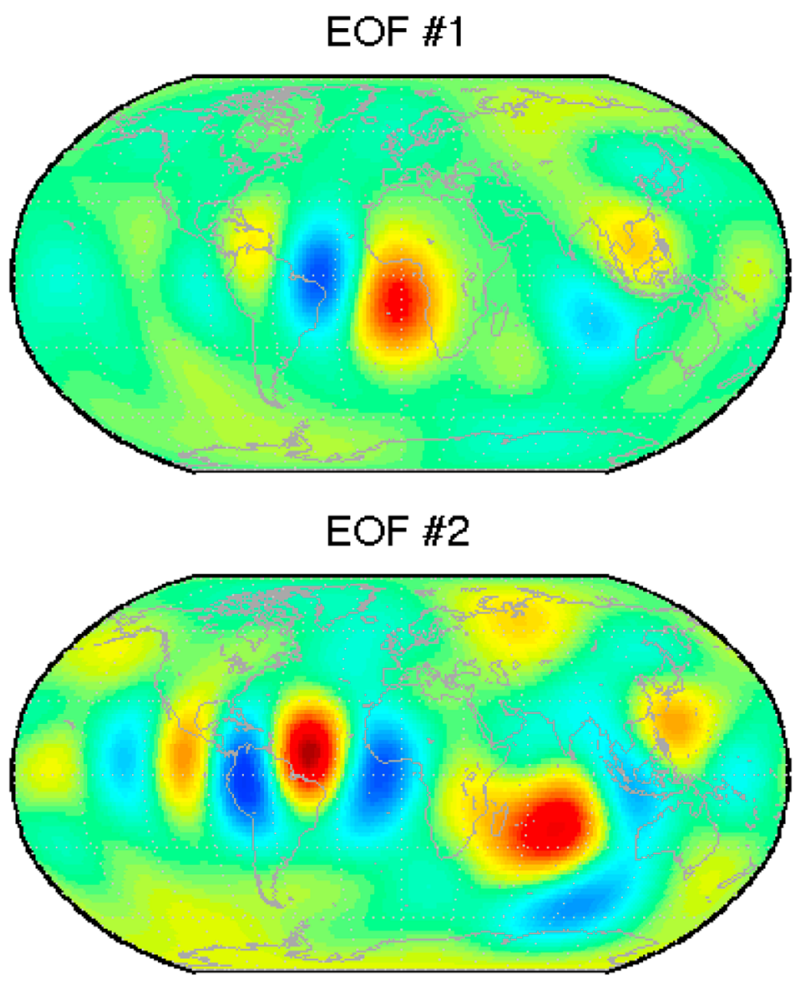

EOF \#3

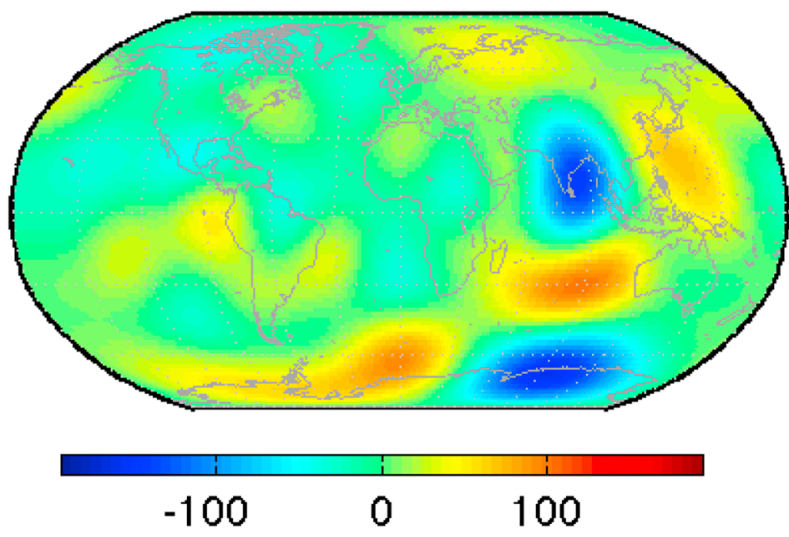

Figure 5. Maps at the core-mantle boundary of the first three empirical orthogonal functions, describing $92 \%$ of the Hann-filtered secular acceleration over 2002.19-2009.51. model epoch and $x$ the coefficient number in the $\mathrm{SH}$ expansion. The product of the obtained $\mathbf{e}_{j}$ empirical orthogonal functions (EOFs) and $\mathbf{a}_{j}$ principal component time series is related to the starting $\mathbf{Z}$ matrix as follows:

$$
z(t, x)=\sum_{j=1}^{p} a_{j}(t) e_{j}(x) .
$$

We found that the first six EOFs describe, respectively, and in decreasing order, $64 \%, 19 \%, 9 \%, 4 \%, 2 \%$, and $1 \%$ of the Hann-filtered SA variance over the time interval 2002.19-2009.51. In total, 99\% of the $S A$ variance is described by the first six EOFs.

As can be seen in Figures 4 and 5, the 2006 and 2009 SA pulses in the Atlantic sector are fully described by the first two EOFs, which account for $83 \%$ of the total SA variance. The first two EOFs peak near 2006 and 2009 and have spatial structures at the $C M B$ very close to that of the pulses, as seen in Figure 2. This is unlike the third EOF, which peaks near 2003 and has its most energetic structures in the Indian Ocean sector. The first EOF displays a half-cycle oscillation, of periodicity about 6 years, while the second EOF displays a full-cycle oscillation, of periodicity about 5 years. Care should be exercised when interpreting principal component periods, especially when periods are close to the length of the record, as EOF analysis will always produce modes that are orthogonal in space and time, event if the true physical modes are not orthogonal [Dommenget and Latif, 2002]. Keeping this cautionary note in mind, we note that the first two EOFs are constructively interfering in 2009, as both the EOFs themselves and their time series are of opposite signs. Near 2006, they peak with a 1 year time difference; thus, their interference is less constructive. These results suggest the existence of a standing wave at the core surface, described by the sum of the first two EOFs.

\section{The 2011 Geomagnetic Jerk}

At the Earth's surface, the SA has a red power spectrum and is dominated by the largest spatial scales. However, despite the geometrical attenuation of the $n=5$ and 6 terms in the SH expansion, the 2006 and 2009 pulses are still detectable in lower degrees, as can be seen in Figure 6 . In fact, except for degree $n=1$, the SA power peaks near 2006 and 2009 for all degrees. It turns out that the power spectrum is red only for degrees larger than $n=4$ and that the power for $n=3$ is most of the time larger than that for $n=1$ and 2, especially during SA pulses. 


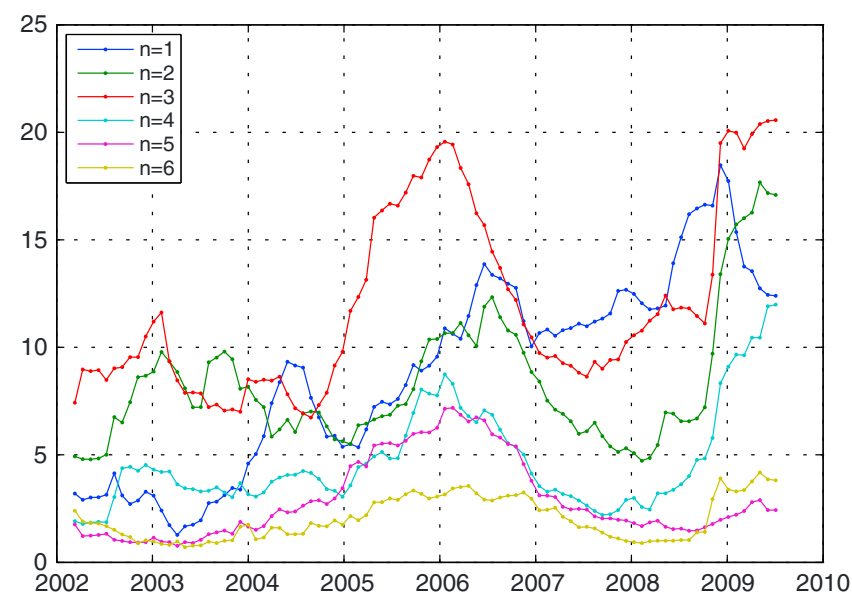

Figure 6. Time variation of the squared secular acceleration power for spherical harmonic degrees $n=1$ to 6 at the Earth's surface. Unit: $\left(n T / y^{2}\right)^{2}$.

beginning or end of SA pulses. Therefore, based upon the maps shown in Figure 7, we expect jerks near 2003, 2007, and after 2009, as the 2009 pulse cannot last for ever. The 2003 and 2007 jerks were already
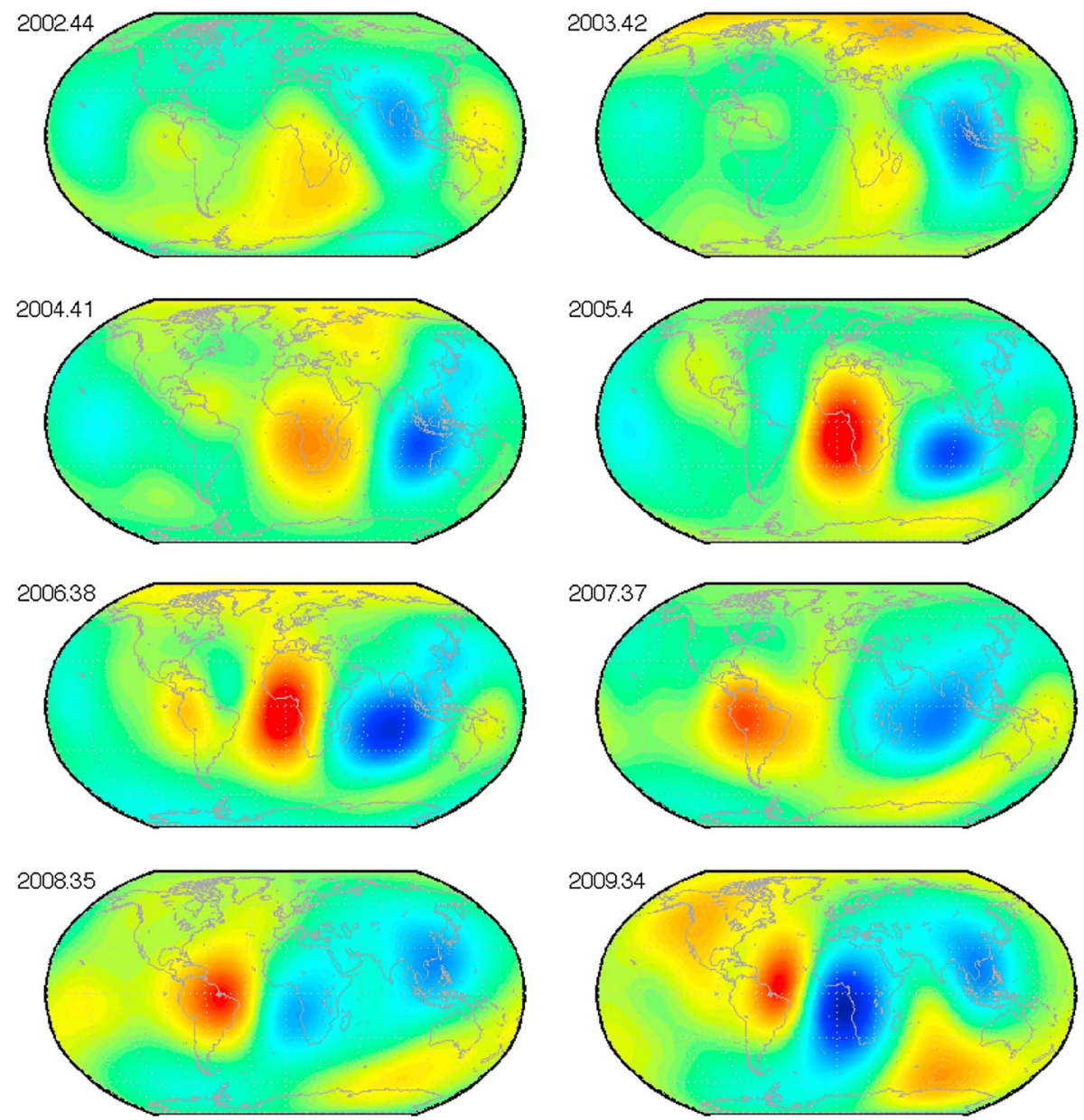

$-15$

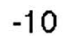

$-5$

0
The 2006 and 2009 pulses are also conspicuous in the SA maps at the Earth's surface (Figure 7). Although much larger than at the CMB, SA patches are located in the same broadly defined regions during pulses, i.e., in the low-latitude Atlantic Ocean and the Indian Ocean. The SA is largest in the Gulf of Guinea for both pulses, and the corresponding patches in 2006 and 2009 are anticorrelated. The differences with respect to Figure 2 are caused by the geometrical attenuation, which is stronger for the smaller patches.

As pointed out in Chulliat et al. [2010], geomagnetic jerks occur at the 

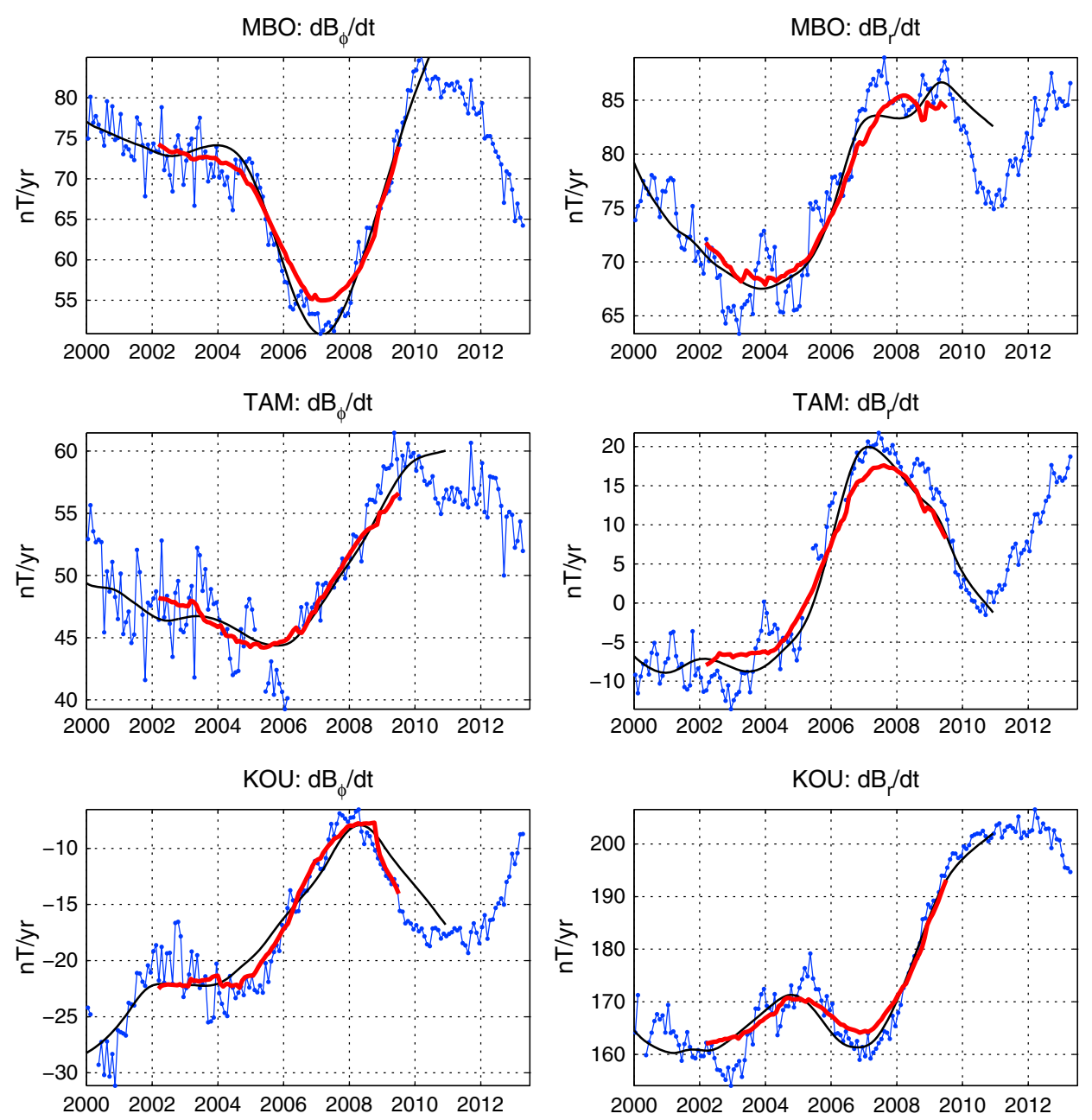

Figure 8. Annual differences (blue dots and curve) of the azimuthal and radial field components at MBO, TAM, and KOU observatories, calculated from improved monthly means (see main text for details). The secular variation calculated from the series of models derived in this work (respectively from the CHAOS-4 model) is represented by thick red lines (respectively thin black lines).

observed and documented in earlier papers. For example, the 2007 jerk was found to be very strong at the MBour (Senegal, International Association of Geomagnetism and Aeronomy (IAGA) code MBO, $14.39^{\circ} \mathrm{N}$, $343.04^{\circ} \mathrm{E}$ ) and Ascension (Ascension Island, IAGA code ASC, $7.9^{\circ} \mathrm{S}, 345.6^{\circ} \mathrm{E}$ ) observatories, as these two observatories are located within the Gulf of Guinea patch in 2006 and 2009 [see Chulliat et al., 2010, Figure 1]. Note that jerks on nonradial components are not maximum in the same locations as jerks on the radial (or Z) component, because the Green function relating the CMB field to the Earth's surface field is not the same for these components; they peak to the north and south, respectively, east and west, of the radial SA patches for the polar (or $X$ ), respectively, azimuthal (or $Y$ ), component.

Recent data provided by observatories of the French Bureau Central de Magnetisme Terrestre network (www.bcmt.fr) reveal that there actually is yet another geomagnetic jerk around 2011, thus marking the end of the 2009 pulse. This can be seen in Figure 8, which shows differences between monthly means at times $t+6$ months and $t-6$ months for the MBour, Tamanrasset (Algeria, IAGA code TAM, $22.79^{\circ} \mathrm{N}$, $5.53^{\circ} \mathrm{E}$ ) and Kourou (French Guiana, IAGA code KOU, $5.21^{\circ} \mathrm{N}, 307.27^{\circ} \mathrm{E}$ ) observatories until May 2013, for the azimuthal and radial components. The north-south component is not shown as it is more noisy due to contamination by external magnetic fields. Annual differences were calculated using definitive observatory data until December 2012 and quasi-definitive data from January 2013 to October 2013. Quasi-definitive data are the data corrected using provisional baselines and produced soon after their acquisition and whose accuracy is expected to be very close to that of definitive data (see International Real-Time Magnetic Observatory Network (INTERMAGNET) Web site, www.intermagnet.org); for observatories like MBO, KOU 


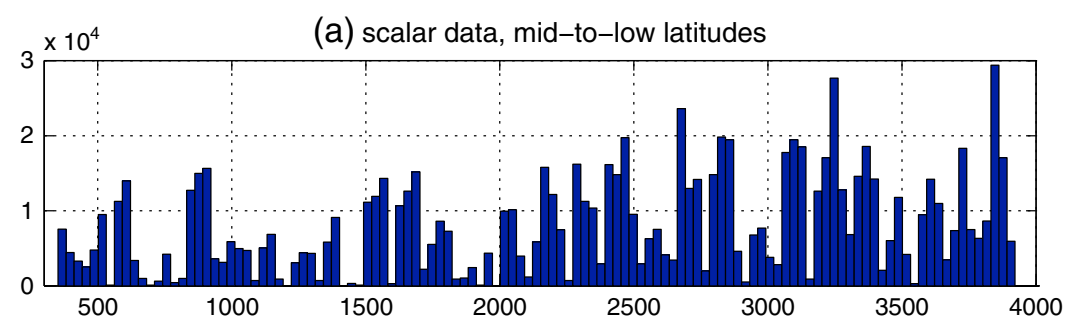

(b) scalar data, north polar latitudes

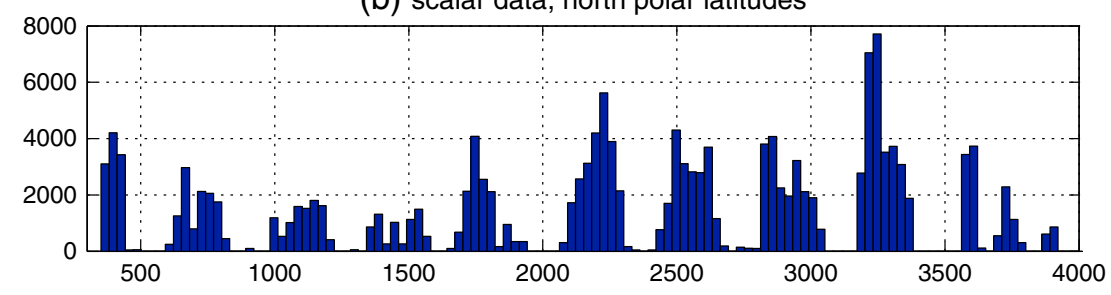

(c) scalar data, south polar latitudes
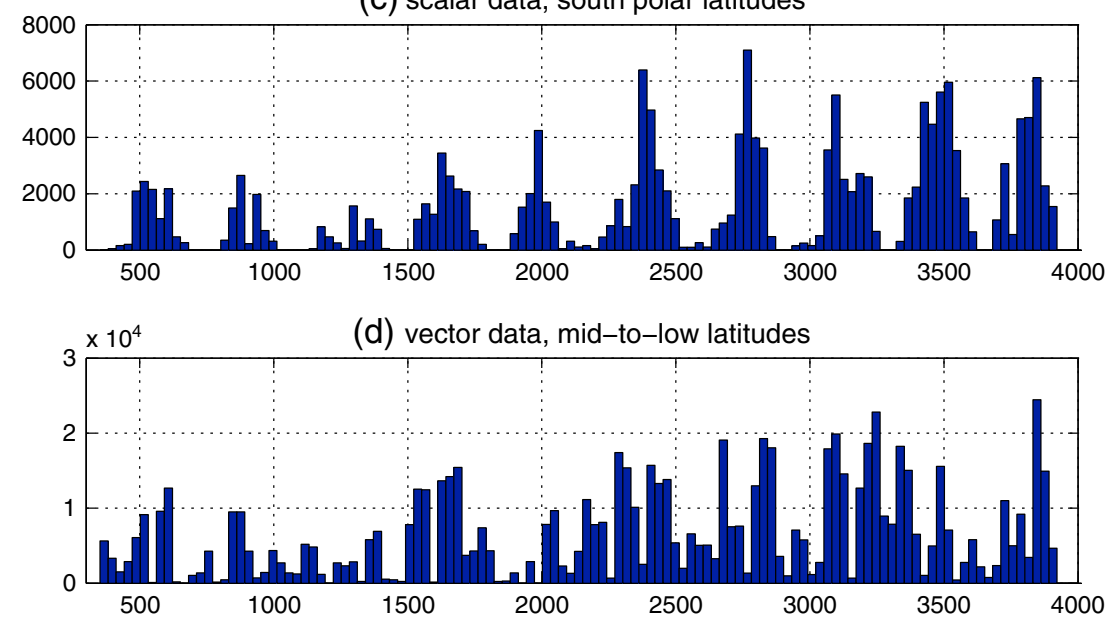

Figure 9. Temporal distributions of the scalar and vector CHAMP data used in the SA modeling. Time in days after 2000. The histogram bin size is 30 days.

and, TAM, quasi-definitive data are typically within $1 \mathrm{nT}$ of the definitive data [Peltier and Chulliat, 2010]. Monthly means were calculated from nightside data (between 22:00 and 5:00 LT) and for geomagnetically quiet time intervals $\left(K_{p}<20,\left|D_{s t}\right|<20 \mathrm{nT}\right)$. At $\mathrm{MBO}$, the jerk is conspicuous on each component, while at TAM, respectively KOU, it is mostly seen on the radial, respectively azimuthal, component. It is worth noting that there is also a jerk near 2011 in Chambon-la-forêt (France, IAGA code CLF, $48.02^{\circ} \mathrm{N}, 2.27^{\circ} \mathrm{E}$ ), mostly on the polar component, and in Addis Ababa (Ethiopia, IAGA code AAE, $9.03^{\circ} \mathrm{N}, 38.77^{\circ} \mathrm{E}$ ), mostly on the radial component.

\section{Discussion}

\subsection{Robustness of Secular Acceleration Pulses}

In this paper, we showed that there were two SA pulses during the past decade, one in 2006 and the other in 2009 , and that the spatial structures of these pulses were anticorrelated at the core surface. The first question to be asked is whether these structures are robust. As explained in section 2, each model is built from 3 years of data, centered on the model epoch. Therefore, the SA in 2006 and 2009 are built from two fully independent data sets. Also, the modeling does not rely on splines for representing the SA time variability and regularization is applied only from degree $n=9$ of the SA. This modeling approach ensures that the low-degree SA is fully determined by the data, whatever the 3 year interval, i.e., even near the boundaries of the decade. This can be seen in Figure 8: at KOU, around 2009, the SV calculated in this work closely follows the curves of observatory annual differences for the azimuthal and radial components, even though these two curves are steep, i.e., the SA is large. Note that the large, positive SA on the radial component 
corresponds to the red patch centered on the mouth of the Amazon River in the 2009.34 map in Figure 7. The CHAOS-4 SV does not follow as closely the azimuthal SV at KOU after 2008, even though CHAOS-4 relies on both CHAMP and observatory data.

The data set itself is of very high quality; CHAMP data have been extensively used and reprocessed several times since the mission launch, and there is very little doubt that most problems with the data have been corrected. In addition, nightside CHAMP data are densely distributed in space and time. Due to CHAMP's slow drift in LT, data at middle to low latitudes are available in intervals of up to 83 days, followed by data gaps of up to 27 days (see Figure 9). Also, data selection leads to some seasonal gaps at polar latitudes, due to the automatic removal of disturbed tracks. However, these gaps are much smaller than the 3 year time interval used for the modeling and their effect is thought to be negligible on the SA modeling. Note that because of the solar minimum, more data are available for modeling at the end of the decade, i.e., at the times of the 2006 and 2009 pulses. This ensures that the SA is well determined for the last window centered in 2009.51, even though CHAMP data are only available until 2010.7.

\subsection{Properties of Geomagnetic Jerks}

SA analysis at the CMB and Earth's surface confirms that geomagnetic jerks in the past decade were caused by the beginning and ending of successive SA pulses at the core surface, as pointed out for the 2003 and 2007 jerks by Chulliat et al. [2010]. Here we showed that another jerk occurred around 2011, following another SA pulse in 2009. The 2007 jerk thus appears to occur between two successive pulses, which could explain its exceptionally large magnitude (about 4 times larger in MBour than the well-known 1979 jerk in Niemegk, Germany).

Properties of geomagnetic jerks have been extensively studied for decades [e.g., Mandea et al., 2010], with some focus on whether or not jerks are global events, and what could be the cause of differential time delays of jerks at different locations [e.g., Pinheiro et al., 2011]. We believe our results bring new insights regarding both questions. First, it is now clear from Figures 2 and 7 that the 2003 and 2007 jerks are not global; their roots are localized SA pulses in the low-latitude Atlantic sector and in the Indian Ocean sector, while their Earth's surface distribution is roughly centered on the same two regions for the vertical component. In the Pacific Ocean sector, the SA at CMB and Earth's surface is close to zero at all times over 2002-2009. Second, SA maps reveal that jerks originating from the same SA pulse beginning or ending may occur at slightly different times at two different locations of the Earth's surface. For example, the beginning of the 2006 pulse is detectable as early as 2003.42 in Southeast Asia, while it only becomes detectable in 2004.41 in Southern Africa (Figure 7). This difference is due to different rise times of the energy of the different spherical harmonic degrees, as can be seen in Figure 1, and to the fact that different combinations of spherical harmonics dominate at different points on the Earth's surface. Jerks observed near 2003 and 2005 were sometimes interpreted as originating from two different events within the core [e.g., Olsen and Mandea, 2008]; however, we showed in this paper that they actually are related to the same SA pulse peaking in 2006. Although differential time delays can be explained by heterogeneities in the SA pulse structure at the core surface, they could also be caused by heterogeneities in the mantle electrical conductivity, or even by a simple 1-D model of mantle electrical conductivity [Pinheiro and Jackson, 2008].

We can continue this line of reasoning: precisely dating the average epoch of a jerk at the global level is probably not very meaningful, as the determination of the time of a jerk at a given point on the Earth's surface depends on the relative location of this point with respect to SA pulse patches at the core surface. If the Earth's radius were to increase by, say, $10 \%$, the determination of the time of each jerk at each observatory would have to be revised, while nothing would have changed within the core. Also, it has long been recognized that jerks occur at different times for different components, as seen in Figure 8. Therefore, we prefer focusing on dating SA pulses at the CMB, rather than jerks at the Earth's surface. The observations reported in the present paper could form the basis of a new, more physically consistent definition of a jerk: provided other jerks prior to 2000 can be similarly related to SA pulses, one could define a jerk as a sudden change of SV occurring at the Earth's surface at the beginning or end of a SA pulse at the CMB.

\subsection{Interpretation of the Observed Standing Wave}

As shown in section 3, the 2006 and 2009 SA pulses have space-time characteristics of a localized standing wave of period about 6 years. The same period of 6 years was recently found by Gillet et al. [2010], when they frequency analyzed the field over the 1955-1985 time interval. They interpreted this wave as being caused by a magnetohydrodynamic (MHD) torsional oscillation within the core, which would then transfer angular 
momentum from the core to the mantle [Jault et al., 1988] and explain a similar 6 year wave in the length of day variation [see, e.g., Holme and de Viron, 2013]. Could the 2006 and 2009 SA pulses also be explained by a torsional oscillation within the core?

Let us consider a standing SA wave of the form

$$
\ddot{B}_{r}(\theta, \phi, t)=\ddot{B}_{r, 0}(\theta, \phi) \cos (\omega t+\psi)
$$

at the core surface, where $\ddot{B}_{r}$ is the radial SA; $\ddot{B}_{r, 0}$ the amplitude of the wave; $\omega=2 \pi / T$ its pulsation; $T=$ 6 years its period; $\theta$ and $\phi$ the colatitude and longitude, respectively; $t$ the time, and $\psi$ the phase for $t=0$. Integrating (2) yields

$$
\dot{B}_{r}(\theta, \phi, t)=\dot{B}_{r, 0}(\theta, \phi)+\frac{\ddot{B}_{r, 0}}{\omega} \sin (\omega t+\psi),
$$

where $\dot{B}_{r}$ is the radial SV and $\dot{B}_{r, 0}$ the average SV over a time interval larger than $T$. Note that $\ddot{B}_{r, 0} \sim 500 \mathrm{nT} / \mathrm{yr}^{2}$ and $\dot{B}_{r, 0} \sim 10^{4} \mathrm{nT} / \mathrm{yr}$ at the core surface; hence, the second term of the right-hand side of equation (3) is about 1 order of magnitude smaller than the first term. Assuming that the frozen flux approximation [Roberts and $S c o t t, 1965]$ is valid on subdecadal time scales, the SV is related to the fluid flow $\mathbf{u}$ at the core surface through

$$
\dot{B}_{r}=-\nabla_{h} \cdot\left(\mathbf{u} B_{r}\right),
$$

where $\nabla_{h}=\boldsymbol{\nabla}-\mathbf{e}_{r} \partial_{r}, \mathbf{e}_{r}$ being the unit radial outward vector. As the signature at the core surface of a torsional oscillation is a zonal toroidal flow, we look for a solution of equation (4) of the form

$$
\mathbf{u}=\mathbf{u}_{0}+\mathbf{u}_{1}
$$

where $\mathbf{u}_{0}$ is a stationary flow and $\mathbf{u}_{1}$ an oscillating, zonal toroidal flow of period $T$. The flow $\mathbf{u}_{1}$ may be written as

$$
\mathbf{u}_{1}=-\mathbf{e}_{r} \times \nabla_{h}\left[\sum_{n \text { odd }} t_{n}^{0}(t) P_{n}^{0}(\cos \theta)\right],
$$

where the $P_{n}^{0}$ are the Schmidt seminormalized Legendre polynomials, and the coefficients $t_{n}^{0}$ are taken for $n$ odd and order zero only, because $\mathbf{u}_{1}$ is purely zonal and symmetric about the equator for torsional oscillations [see, e.g., Dumberry and Bloxham, 2004]. Substituting (5) into (3) and extracting the zeroth- and first-order terms yields

$$
\begin{gathered}
\dot{B}_{r, 0}=-\nabla_{h} \cdot\left(\mathbf{u}_{0} B_{r, 0}\right), \\
\frac{\ddot{B}_{r, 0}}{\omega} \sin (\omega t+\psi)=-\nabla_{h} \cdot\left(\mathbf{u}_{1} B_{r, 0}\right),
\end{gathered}
$$

where $B_{r, 0}$ is the average MF at the core surface over a time interval larger than $T$. Taking $t_{n}^{0}(t)=\bar{t}_{n}^{0} \sin (\omega t+\psi)$ and substituting (6) into (8) then leads to

$$
\frac{\ddot{B}_{r, 0}}{\omega}=\left[\sum_{n \text { odd }} \bar{t}_{n}^{0} \frac{\partial P_{n}^{0}(\cos \theta)}{\partial \theta}\right] \frac{1}{\sin \theta} \frac{\partial B_{r, 0}}{\partial \phi} .
$$

We solved equation (9) for the first four $\bar{t}_{n}^{0}$ coefficients (i.e., until $n=7$ ), using standard least squares inversion on a $2^{\circ} \times 2^{\circ}$ grid at the CMB and taking the first EOF (see section 3 ) as the SA wave amplitude. We found that less than $10 \%$ of the signal could actually be fitted by these four coefficients. This shows that a zonal toroidal flow cannot explain a 6 year SA wave of the form (2) over the past decade. Thus, it is highly unlikely that the observed 2006 and 2009 SA pulses could be caused by a torsional oscillation.

Slow magneto-Coriolis (MC) waves are another type of MHD waves within the core that could explain the observed 6 year periodicity of the SA. Such waves have been invoked to explain the observed westward motion of flux patches in the equatorial region over the past 400 years [Finlay and Jackson, 2003]. They could have periodicities ranging from 1 year to several thousands of years, depending on the wave 
number and the strength of the toroidal field within the core [Finlay et al., 2010]. The difficulty with this interpretation lies in the large toroidal field that would be needed to sustain a MC wave at subdecadal frequencies; for example, according to theory, a MC wave of wave number $m=8$ would require a toroidal field strength of 10-100 mT within the core [see, e.g., Finlay et al., 2010, Figure 4], i.e., about 100 times the strength of the poloidal field at the core surface. Interestingly, this value would actually be in agreement with recent estimates of the toroidal field based upon the analysis of torsional oscillations within the core [Gillet et al., 2010].

Another possible interpretation of a subdecadal wave at the core surface would rely on the hypothesis made by Braginsky [1999] and recently supported by seismic observations [Helffrich and Kaneshima, 2010, 2013] that there could be a thin (a few tens of kilometers), stably stratified layer at the top of the core. This layer would have formed as a result of the accumulation at the top of the core of light elements released during the differential crystallization of the inner core. Braginsky [1999] theoretically investigated MHD waves within such a layer and found magnetic Rossby waves with subdecadal periods. Further investigations would however be needed to determine if such waves could actually explain the space time characteristics of the 2006 and 2009 SA pulses.

\section{Conclusion}

In this paper, we derived MF, SV, and SA spherical harmonic models every 30 days on a 3 year sliding window centered on epochs varying from 2002.19 to 2009.51. To reduce artifacts and spurious signals in the obtained SA, we used a homogeneous CHAMP data set; corrected data using the latest models accounting for other sources did not regularize SA degrees lower than 9 and used a second-order Taylor expansion in time instead of more sophisticated temporal basis functions such as splines. Time variations of the degree-by-degree power spectra revealed that the SA underwent two power pulses, centered in 2006 and 2009. These pulses are seen both at the CMB (mostly at degrees 5 and 6 ) and at the Earth's surface (mostly at degrees 2 and 3). They are localized in two areas: the low-latitude Atlantic sector and the Indian sector. Due to differential geometrical attenuation, the 2006 and 2009 pulses are of smaller spatial extent at the CMB than at the Earth's surface.

We found that the spatial features of the 2006 and 2009 pulses at the CMB in the Atlantic sector were markedly anticorrelated. A standard time-longitude representation suggests that this pattern is that of a standing wave at the core surface. This interpretation is also supported by an EOF analysis of the SA over the 2002-2009.5 time interval: most of the SA signal can be described as the superposition of two waves of 5 and 6 year periods, simultaneously peaking in 2009.

The 2006 and 2009 pulses are separated by the 2007 geomagnetic jerk. This jerk is of exceptionally large amplitude in the Atlantic sector, i.e., where the pulses are strongest at the CMB. Two other jerks occurred in the past decade: one at the beginning of the 2006 pulse, in the time interval 2003 to 2005, depending on the location at the Earth's surface, and the other at the ending of the 2009 pulse, near 2011. More observatory data for years 2012 and 2013 will be needed to properly describe this latest jerk, but there is no doubt a jerk occurred after 2010 in observatories close to the low-latitude Atlantic sector. These results support the idea (expressed in Chulliat et al. [2010]) that at least some jerks observed at the Earth's surface result from the beginning and ending of SA pulses at the core surface. As regard the 2003, 2007, and 2011 jerks, these seem to be caused by a standing wave within the core.

The origin of the observed standing wave still remains to be elucidated. We showed that zonal toroidal flows alone could only account for less than $10 \%$ of the wave. This is quite intriguing as torsional oscillations were recently found by Gillet et al. [2010] to account for a 6 year periodic signal observed in the MF over the 1955-1985 time interval. Other possible 6 year MHD waves in the core include a slow MC wave and a magnetic Rossby wave in a stratified layer near the core surface. The existence of either of these two waves with period around 6 years would have significant implications regarding our understanding of core dynamics and the geodynamo: either the strength of the toroidal field would have to be reevaluated up to 10-100 mT (slow MC wave) or a stably stratified layer at the top of the core would have to be assumed (magnetic Rossby wave). We anticipate that the Swarm mission [Friis-Christensen et al., 2006], recently launched in Fall 2013, will provide further constraints on the spatial and temporal characteristics of the wave reported in this paper and thus contribute to its elucidation. 


\section{Acknowledgments}

The CHAMP mission is supported by the German Aerospace Center (DLR) and the Federal Ministry of Education and Research. The results presented in this paper rely on data collected at magnetic observatories. We thank the national institutes that support them and INTERMAGNET for promoting high standards of magnetic observatory practice (www.intermagnet.org). We thank two reviewers for constructive comments. The research reported here was financially supported by CNES and by the Cooperative Institute for Research in Environmental Sciences, University of Colorado Boulder. This is IPGP contribution 3496

\section{References}

Bloxham, J., and A. Jackson (1992), Time-dependent mapping of the magnetic field at the core-mantle boundary, J. Geophys. Res., 97, $19,537-19,563$.

Braginsky, S. I. (1999), Dynamics of the stably stratified ocean at the top of the core, Phys. Earth Planet. Inter., 111, 21-34.

Chulliat, A., E. Thébault, and G. Hulot (2010), Core field acceleration pulse as a common cause of the 2003 and 2007 geomagnetic jerks, Geophys. Res. Lett., 37, L07301, doi:10.1029/2009GL042019.

Courtillot, V., J. Ducruix, and J.-L. Le Mouël (1978), Sur une accélération récente de la variation séculaire du champ magnétique terrestre, C. R. Acad. Sci. D, 287, 1095-1098.

Dommenget, D., and M. Latif (2002), A cautionary note on the interpretation of EOFs, J. Clim., 15, 216-225.

Dumberry, M., and J. Bloxham (2004), Variations in the Earth's gravity field cause by torsional oscillations in the core, Geophys. J. Int., 159, 417-434.

Finlay, C. C., and A. Jackson (2003), Equatorially dominated magnetic field change at the surface of Earth's core, Science, 300, 2084-2086.

Finlay, C. C., M. Dumberry, A. Chulliat, and M. A. Pais (2010), Short timescale core dynamics: Theory and observations, Space Sci. Rev., 155 $177-218$.

Finlay, C. C., A. Jackson, N. Gillet, and N. Olsen (2012), Core surface magnetic field evolution 2000-2010, Geophys. J. Int., 189, 761-781.

Friis-Christensen, E., H. Lühr, and G. Hulot (2006), Swarm: A constellation to study the Earth's magnetic field, Earth Planets Space, 58, 351-358.

Gillet, N., D. Jault, E. Canet, and A. Fournier (2010), Fast torsional waves and strong magnetic field within the Earth's core, Nature, 465 , 74-77.

Helffrich, G., and S. Kaneshima (2010), Outer-core compositional stratification from observed core wave speed profiles, Nature, 468 $807-810$

Helffrich, G., and S. Kaneshima (2013), Causes and consequences of outer core stratification, Phys. Earth Planet. Inter., 223, 2-7.

Holme, R., and O. de Viron (2013), Characterization and implications of intradecadal variations in length of day, Nature, 499, $202-204$.

Jault, D., C. Gire, and J.-L. Le Mouël (1988), Westward drift, core motions and exchanges of angular momentum between core and mantle, Nature, 333, 353-356.

Korte, M., and C. G. Constable (2005), Continuous geomagnetic field models for the past 7 millennia: 2. CALS7K, Geochem. Geophys. Geosyst., 6, Q02H16, doi:10.1029/2004GC000801.

Kuvshinov, A., and N. Olsen (2005), 3-D modelling of the magnetic fields due to ocean tidal flow, in Earth Observation With CHAMP, Results From Three Years in Orbit, edited by C. Reigber et al., pp. 359-365, Springer, Berlin, Germany.

Lesur, V., I. Wardinski, M. Hamoudi, and M. Rother (2010), The second generation of the GFZ reference internal magnetic model: GRIMM-2, Earth Planets Space, 62, 765-773.

Lühr, H., and S. Maus (2010), Solar cycle dependence of quiet-time magnetospheric currents and a model of their near-Earth magnetic fields, Earth Planets Space, 62, 843-848.

Lühr, H., M. Rother, S. Maus, W. Mai, and D. Cooke (2003), The diamagnetic effect of the equatorial Appleton anomaly: Its characteristics and impact on geomagnetic field modeling, Geophys. Res. Lett., 30, 1906, doi:10.1029/2003GL017407.

Mandea, M., R. Holme, A. Pais, K. Pinheiro, A. Jackson, and G. Verbanac (2010), Geomagnetic jerks: Rapid core field variations and core dynamics, Space Sci. Rev., 155, 147-175.

Maus, S., M. Rother, C. Stolle, W. Mai, S. Choi, H. Lühr, D. Cooke, and C. Roth (2006), Third generation of the Potsdam Magnetic Model of the Earth (POMME), Geochem. Geophys. Geosyst., 7, Q07008, doi:10.1029/2006GC001269.

Maus, S., C. Manoj, J. Rauberg, I. Michaelis, and H. Lühr (2010), NOAA/NGDC candidate models for the 11 th generation International Geomagnetic Reference Field and the concurrent release of the 6th generation POMME magnetic model, Earth Planets Space, 62, 729-735.

Olsen, N., and M. Mandea (2007), Investigation of a secular variation impulse using satellite data: The 2003 geomagnetic jerk, Earth Planet. Sci. Lett., 255, 94-105.

Olsen, N., and M. Mandea (2008), Rapidly changing flows in the Earth's core, Nat. Geosci., 1, 390-394.

Olsen, N., M. Mandea, T. J. Sabaka, and L. Tøffner-Clausen (2009), CHAOS-2-A geomagnetic field model derived from one decade of continuous satellite data, Geophys. J. Int., 179, 1477-1487.

Olsen, N., M. Mandea, T. J. Sabaka, and L. Tøffner-Clausen (2010), The CHAOS-3 geomagnetic field model and candidates for the 11 th generation IGRF, Earth Planets Space, 62, 719-727.

Peltier, A., and A. Chulliat (2010), On the feasibility of promptly producing quasi-definitive magnetic observatory data, Earth Planets Space, 62, e5-e8, doi:10.5047/eps.2010.02.002.

Pinheiro, K., and A. Jackson (2008), Can a 1-D mantle electrical conductivity model generate magnetic jerk differential time delays?, Geophys. J. Int., 173, 781-792.

Pinheiro, K., A. Jackson, and C. C. Finlay (2011), Measurements and uncertainties of the occurrence time of the 1969, 1978, 1991, and 1999 geomagnetic jerks, Geochem. Geophys. Geosyst., 12, Q10015, doi:10.1029/2011GC003706.

Preisendorfer, R. W. (1988), Principal Component Analysis in Meteorology and Oceanography, 436 pp., Elsevier, Amsterdam, Netherlands Roberts, P. H., and S. Scott (1965), On analysis of the secular variation, J. Geomag. Geoelectr., 17, 137-151.

Silva, L., L. Jackson, and J. Mound (2012), Assessing the importance and expression of the 6 year geomagnetic oscillation, J. Geophys. Res., 117, B10101, doi:10.1029/2012JB009405. 